\title{
Discovery of a bright X-ray transient in the Galactic Center with XMM-Newton
}

\author{
D. Porquet ${ }^{1}$, N. Grosso ${ }^{2}$, V. Burwitz ${ }^{1}$, I. L. Andronov ${ }^{3,4}$, B. Aschenbach ${ }^{1}$, P. Predehl ${ }^{1}$, and R. S. Warwick ${ }^{5}$ \\ 1 Max-Planck-Institut für extraterrestrische Physik, PO Box 1312, Garching bei München 85741, Germany \\ e-mail: dporquet@mpe.mpg.de \\ ${ }^{2}$ Laboratoire d'Astrophysique de Grenoble, Université Joseph-Fourier, BP 53, 38041 Grenoble Cedex 9, France \\ 3 Department of Astronomy, Odessa National University, T. G. Shevchenko park, 65014 Odessa, Ukraine \\ 4 Crimean Astrophysical Observatory, 98409 Nauchny, Ukraine \\ 5 Department of Physics and Astronomy, University of Leicester, Leicester LE1 7RH, UK
}

Received 10 September 2004 / Accepted 2 December 2004

\begin{abstract}
We report the discovery of a bright X-ray transient object, XMMU J174554.4-285456, observed in outburst with XMM-Newton on October 3, 2002, and located at 6.3' from Sgr A*, the supermassive black hole at the Galactic center. This object exhibits a very large X-ray luminosity variability of a factor of about 1300 between two X-ray observations separated by four months. The $\mathrm{X}$-ray spectrum is best fitted by a power-law with a photon index of $1.6 \pm 0.2$ and absorption column density of $14.1_{-1.4}^{+1.6} \times 10^{22} \mathrm{~cm}^{-2}$. This large absorption suggests this source is located at the distance of the Galactic center, i.e., $8 \mathrm{kpc}$. The $2-10 \mathrm{keV}$ luminosity is about $1.0 \times 10^{35}(\mathrm{~d} / 8 \mathrm{kpc})^{2} \mathrm{erg} \mathrm{s}^{-1}$. A pulsation period of about $172 \mathrm{~s}$ is hinted by the timing analysis. The X-ray properties strongly suggest a binary system with either a black hole or a neutron star for the compact object.
\end{abstract}

Key words. Galaxy: center - X-rays: binaries - X-rays: individuals: XMMU J174554.4-285456

\section{Introduction}

The Galactic center region $\left(\Delta l \sim 2^{\circ}, \Delta b \sim 0.5^{\circ}\right)$ is very complex in X-rays with both diffuse emission and point-like sources, counterparts of fluorescent molecular clouds, supernova remnants, compact objects, and stellar clusters. In addition to Sgr A*, the supermassive black hole at the Galactic center, this region shelters accreting compact objects, such as neutron stars and black hole candidates (e.g., Churazov et al. 1997; Sidoli et al. 1999; Sakano et al. 2002; Porquet et al. 2003a), which can be transient sources in X-rays. Therefore, repeated X-ray observations of this region, achieved for the monitoring of $\mathrm{Sgr} \mathrm{A}^{*}$, give us the opportunity to catch one of these compact objects during an outburst phase, and to shed light on their nature. We report here the serendipitous discovery on October 3, 2002 with XMM-Newton of a bright X-ray transient source located at 6.3' from Sgr A*.

\section{XMM-Newton observation}

The XMM-Newton observation is the one obtained on October 3, 2002, where a very bright X-ray flare was reported from Sgr A* (Porquet et al. 2003b). The observation exposure times are $\sim 16.8 \mathrm{ks}$ and $\sim 13.9 \mathrm{ks}$ for the MOS and PN cameras, respectively. The data were processed with SAS (version 6.0). X-ray events with patterns $0-12$ and $0-4$ are used

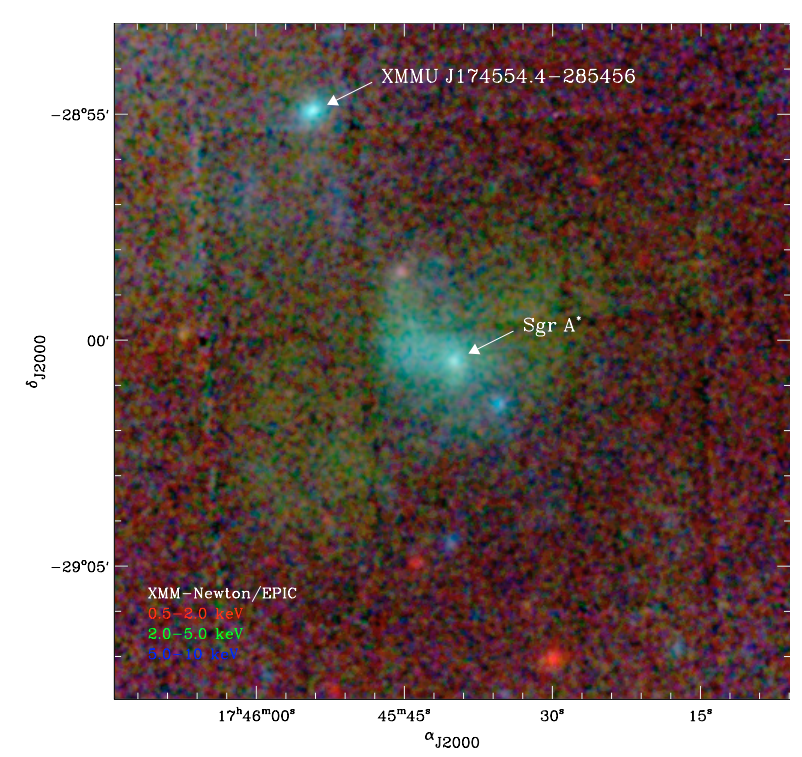

Fig. 1. XMM-Newton/EPIC image of the bright X-ray transient XMMU J174554.4-285456 close to Sgr A*. Red, green and blue code for photons with energy $0.5-2,2-5$, and 5-10 keV, respectively.

for MOS and PN, respectively. We select only events with data quality flag equal to 0 . The astrometry is the one reported in Porquet et al. (2003b). Figure 1 shows the $7^{\prime} \times 7^{\prime}$ central part of 


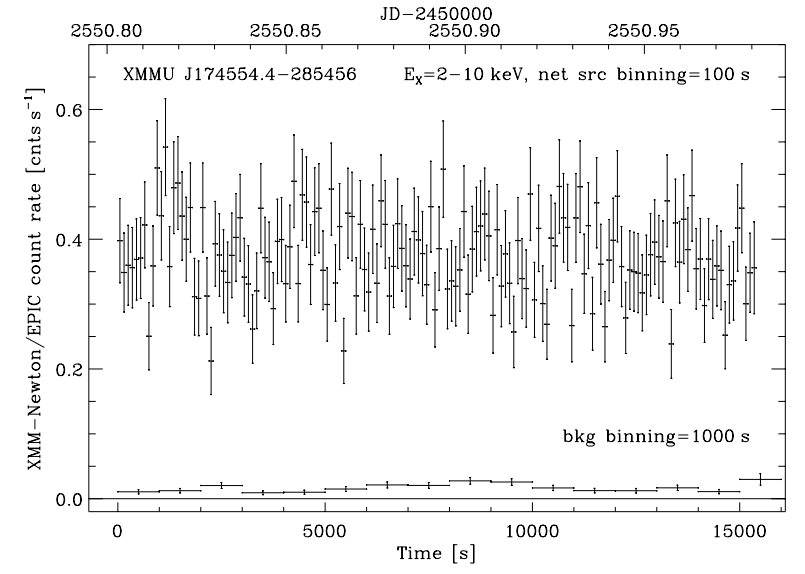

Fig. 2. XMM-Newton/EPIC background subtracted light curve of XMMU J174554.4-285456 in the 2-10 keV energy band. The lower light curve shows the background level scaled to the source extraction area.

this observation in the $0.5-10 \mathrm{keV}$ energy range: a new $\mathrm{X}$-ray source, as bright as $\mathrm{Sgr} \mathrm{A}^{*}$, is clearly seen at 6.3' from Sgr A*. Its position in $\mathrm{J} 2000$ coordinates is $\alpha=17 \mathrm{~h} 45 \mathrm{~m} 54.4 \mathrm{~s}, \delta=$ $-28^{\circ} 54^{\prime} 56^{\prime \prime}$ with a $90 \%$ confidence level error position of $2.3^{\prime \prime}$ in radius. We name this new X-ray source XMMU J174554.4285456. We did not find any known counterpart of this source in the SIMBAD data base. We note that this area was previously observed in X-rays with higher sensitivity both with XMM-Newton (e.g., Sakano et al. 2004) and Chandra (e.g., Muno et al. 2003), and that this source was not detected. In addition, during the Chandra observation on June 19, 2003, about eight months after the present observation, the source was not detected, hence XMMU J174554.4-285456 is a transient X-ray source.

We extract the source events from a circular region of $12^{\prime \prime}$-radius centered on the X-ray source position, and the background events from a rectangular region free of X-ray sources lying on the same CCD and close to the transient. Photon arrival times were computed for the solar system barycenter. Figure 2 displays the background subtracted EPIC light curve of XMMU J174554.4-285456 in the 2-10 keV energy band, during the time interval when the three cameras were observing together. We do not see any obvious variations of the light curve as type-I burst, or eclipse.

\section{Spectral analysis}

We use for the spectral analysis only PN data, which have a higher $\mathrm{S} / \mathrm{N}$ and better energy coverage than the MOS data. The spectrum is binned to a minimum of 20 counts per bin. The fitting parameter errors quoted correspond to $90 \%$ confidence ranges for one interesting parameter $\left(\Delta \chi^{2}=2.71\right)$. In the following, we use the updated X-ray absorption cross-sections and abundances of the interstellar medium (ISM) of Wilms et al. (2000). The PN spectrum, shown in Fig. 3, is strongly absorbed below $2 \mathrm{keV}$, evidence of a large absorption by the ISM along the line-of-sight. We fit the data taking into account the scattering of X-rays by dust, using the scatter model (Predehl \& Schmitt 1995) assuming a visual extinction value

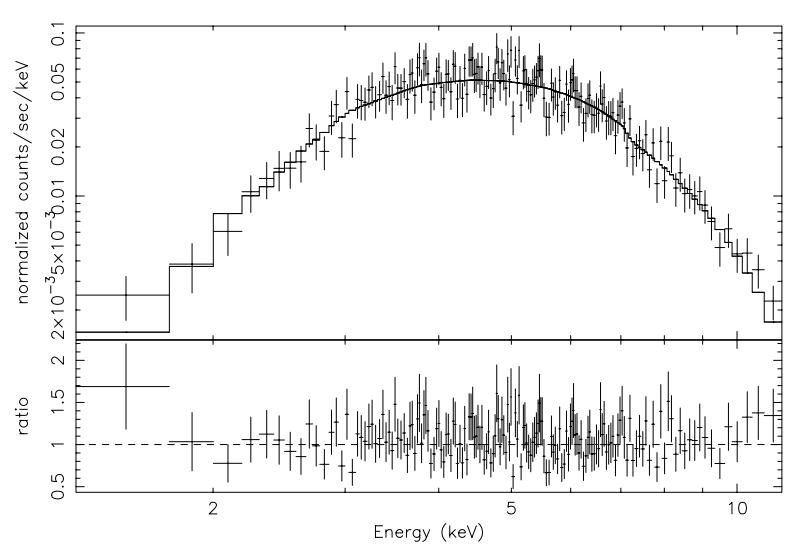

Fig. 3. PN spectrum of XMMU J174554.4-285456 with the best fit model using an absorbed power-law continuum (see Table 1) and datato-model ratio.

Table 1. Models of the PN spectrum of XMMUJ174554.4285456, taking into account absorption. po: power law. bb: black body. diskbb: multi black body (Mitsuda et al. 1984). brems: bremsstrahlung. The number of degrees of freedom is 161 . The column density is expressed in units of $10^{22} \mathrm{~cm}^{-2}$. The flux $\left(F_{\mathrm{X}}\right)$ and luminosity $\left(L_{\mathrm{X}}\right)$ corrected for absorption are expressed in $10^{-11} \mathrm{erg} \mathrm{cm}^{-2} \mathrm{~s}^{-1}$ and $10^{35} \mathrm{erg} \mathrm{s}^{-1}$, respectively. The luminosity is calculated assuming $d=8 \mathrm{kpc}$.

\begin{tabular}{cccccccc}
\hline \hline Model & $\mathcal{N}_{\mathrm{H}}$ & $\Gamma$ or $k T$ & $\chi_{\text {red }}^{2}$ & $F_{\mathrm{X}}$ & $L_{\mathrm{X}}$ & $F_{\mathrm{X}}$ & $L_{\mathrm{X}}$ \\
& & & & & $2-10 \mathrm{keV}$ & $0.5-10 \mathrm{keV}$ \\
\hline po & $14.1_{-1.4}^{+1.6}$ & $1.65_{-0.16}^{+0.17}$ & 0.87 & 1.4 & 1.0 & 2.1 & 1.5 \\
bb & $7.3 \pm 0.9$ & $1.9 \pm 0.1$ & 1.08 & 1.0 & 0.7 & 1.0 & 0.7 \\
diskbb & $11.0 \pm 1.0$ & $3.7 \pm 0.5$ & 0.93 & 1.2 & 0.9 & 1.4 & 1.0 \\
brems & $13.6 \pm 1.2$ & $22_{-15}^{+40}$ & 0.88 & 1.3 & 0.9 & 1.8 & 1.3 \\
\hline
\end{tabular}

$A_{\mathrm{V}}=30 \mathrm{mag}$, as determined from IR observations of stars close to Sgr A* (e.g., Rieke et al. 1989).

The PN spectrum is well fitted by the standard absorbed continuum models, such as power-law, black-body, multi black body, and bremsstrahlung (Table 1). However, the temperatures found for the latter three models are rather high, and the spectrum is not well fitted above $10 \mathrm{keV}$ by the black-body and the multi black body models. According to Predehl \& Schmitt (1995), $A_{\mathrm{V}}=30$ mag corresponds to an hydrogen column density of about $6 \times 10^{22} \mathrm{~cm}^{-2}$ towards the Galactic center located at about $8 \mathrm{kpc}$. The updated ISM abundance used here lead to absorption column density values about $30 \%$ larger than those derived assuming solar abundance as in Predehl \& Schmitt (1995). For comparison we found for Sgr A* (during its X-ray flare) a column density of about Therefore, the hydrogen column density estimated here for the absorbed power law model is consistent with a location of this transient object at the distance of the Galactic center. We also test a thin thermal model mekal but in case of solar metal abundance, we obtain an unphysically high temperature greater than $50 \mathrm{keV}$. If the metal abundance is let as a free parameter, the temperature is lower with $k T=18.6_{-7.0}^{+22.8} \mathrm{keV}$, and the upper limit of the metal abundance is about 0.3 , due mainly to the lack of obvious spectral emission lines. Therefore the power-law model is our best fit. 

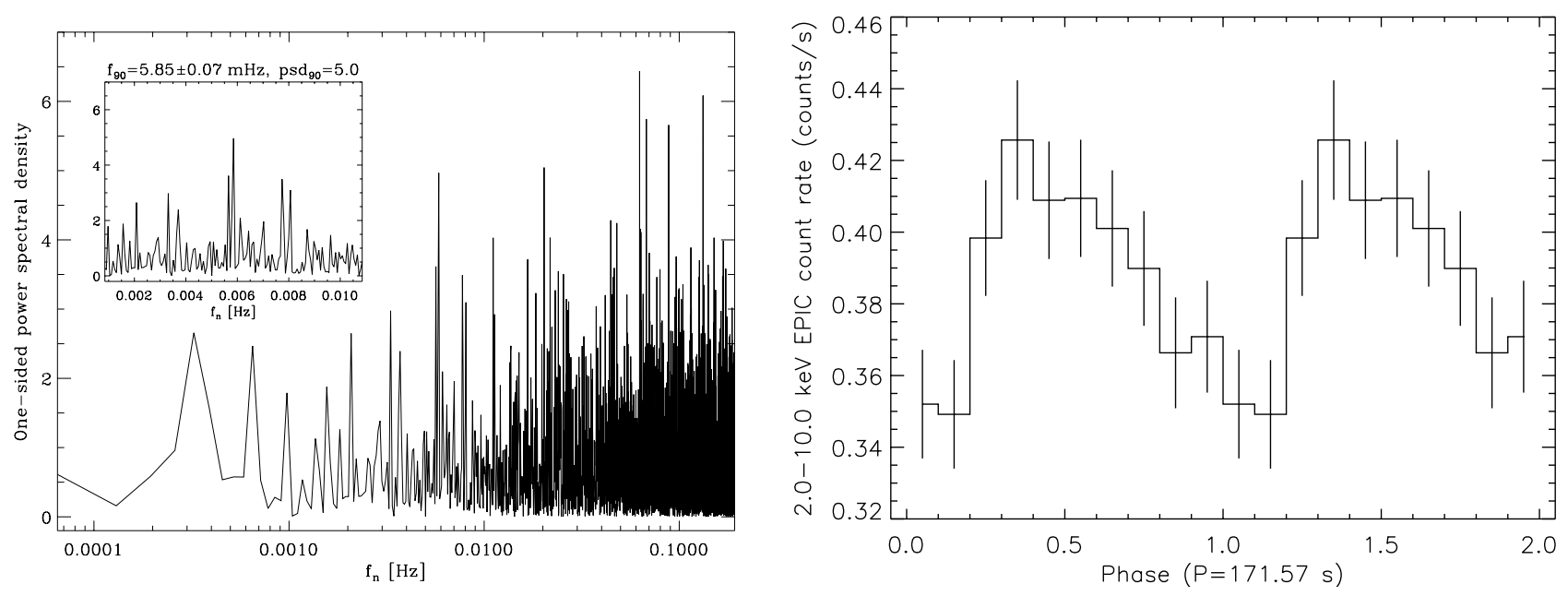

Fig. 4. Timing analysis of XMMU J174554.4-285456 in the 2-10 keV energy band. left: power spectrum of the EPIC 2.6 s-binned light curve. The enlargement shows the X-ray pulsation candidate at the frequency $5.85 \pm 0.07 \mathrm{mHz}$ corresponding to a period of $171 \pm 2 \mathrm{~s}$. right: folded light curve for the period $171.6 \pm 0.3 \mathrm{~s}$ found with periodogram analysis.

We obtain an upper limit of the equivalent widths for a narrow iron $\mathrm{K} \alpha$ line $(\sigma=10 \mathrm{eV})$ between 6.4 ("neutral") and $7 \mathrm{keV}$ (H-like) of 30-86 eV. To look for a possible time variability of the spectrum, we split the observation into two parts, but we find no statistically significant variations of the photon index, the absorption column and the $2-10 \mathrm{keV}$ flux. $20 \times 10^{22} \mathrm{~cm}^{-2}$ (Porquet et al. 2003b).

\section{Period analysis}

We have searched the combined EPIC data with a time bin of $2.6 \mathrm{~s}$ for X-ray pulsations in the $2-10 \mathrm{keV}$ range. The FFT power density spectrum shows a peak at $5.85 \pm 0.07 \mathrm{mHz}$ or $171 \pm 2 \mathrm{~s}$ (Fig. 4, left). The probability that the peak at this frequency is produced by just white photon noise is $2 \%$ has been found by Monte Carlo simulations. To cope with the possibility that the signal is not constant with just random fluctuations, we assessed the false alarm probability making use of the periodogram analysis (Lomb 1976, Scargle's 1982) in the form suggested by Andronov (1994): $x(t)=a-R \cos \left(2 \pi\left(t-T_{0}\right) / P\right)$, where $x(t)$ is the $2.6 \mathrm{~s}$-binned signal at time $t, a$ is phaseaveraged mean value, $R$ is the semi-amplitude, $P$ is the trial period, and $T_{0}$ is an initial epoch. The MOS1, MOS2 and PN data as well as their sum have been analysed separately, and a candidate period at $P=171.57 \pm 0.25 \mathrm{~s}$ has been found consistent with the period found by the FFT analysis. The candidate period has a signal to noise ratio $(\mathrm{S} / \mathrm{N})$ of 2.9 (MOS1), 3.3 (MOS2), 6.0 (PN) and 9.7 (total). The $\mathrm{S} / \mathrm{N}$ ratio is defined as the ratio of the count rate obtained for the candidate frequency divided by the count rate averaged over the full frequency band. Monte Carlo simulations were done for 50000 evenly spaced trial frequencies ranging from $10^{-5} \mathrm{~s}^{-1}$ up to the Nyquist frequency of $1 / 2 \mathrm{~s}^{-1}$, and resulted in a false alarm probability of 0.37 for 7638 independent frequencies. We have also exercised a test on the phase relation among the three instruments by comparing the fitted values for $T_{0}$, which are $T_{0, \mathrm{MOS} 1}=7678 \pm 11 \mathrm{~s}, T_{0, \mathrm{MOS} 2}=7682 \pm 11 \mathrm{~s}$, and
$T_{0, \mathrm{PN}} 7665 \pm 8 \mathrm{~s}$. The largest phase shift is $0.10 \pm 0.08$ which suggests that there is no significant shift. In the context of this approach, we have estimated a false alarm probability, defined as the probability to get by chance the observed phase "coincidence", by computing $10^{8}$ trial data sets with uniformly distributed phases. For each trial set, a weighted mean and variance is computed using the previous measured instrumental uncertainties. The simulated variance of the phases exceeds the measured variance in $3.4 \%$ of all trials. In summary the false alarm probability for the suggested period is somewhere between 2 and $3.4 \%$ ignoring any modulation of the signal and could be around $37 \%$ otherwise. Finally, we give the best fit parameters of the signal function for the data of the three instruments combined: $a=0.368 \pm 0.005, R=0.032 \pm 0.007$, $T_{0}=7672.2 \pm 6.2 \mathrm{~s}$ (corresponding to $\mathrm{mjd}=2550.80667$, i.e. October 3, 2002 at 7:21:36.0). The peak-to-peak amplitude of the periodic variations is $\sim 16 \% \pm 2$, which is the same for all three instruments within the error estimates. Figure 4 (right) shows the light curve folded with this period. We have extracted phase resolved spectra selecting data corresponding to peak (phase $=0.2-0.6$ ), and to trough $($ phase $=0.6-1.2)$, and we found no significant spectral differences.

\section{The nature of XMMU J174554.4-285456}

We estimate the amplitude of the luminosity outburst from the deepest Chandra observation of Sgr A*, $167 \mathrm{ks}$ of exposure obtained on May 25, 2002, i.e. four months before our XMM-Newton observation. We found a 3- $\sigma$ count rate upper limit of $1.2 \times 10^{-4}$ count $^{-1}$ at the location of XMMU J174554.4-285456 in the 0.5-10 keV energy band. Assuming $\Gamma=1.6$ (see Table 1), we find $L_{\mathrm{X}}(0.5-10 \mathrm{keV}) \leq 1.2 \times 10^{32}(\mathrm{~d} / 8 \mathrm{kpc})^{2} \mathrm{erg} \mathrm{s}^{-1}$ during the quiescent state. Therefore, this source has exhibited a luminosity increase of a factor of about 1300 over four months. In addition the source was not detected eight months after the present XMM-Newton observation by Chandra during a 
short exposure (25 ks) obtained on June 19, 2003 leading to a $3-\sigma 0.5-10 \mathrm{keV}$ count rate upper limit of $3.7 \times 10^{-4}$ count s $^{-1}$ at the location of XMMU J174554.4-285456, corresponding to $L_{\mathrm{X}}(0.5-10 \mathrm{keV}) \leq 3.7 \times 10^{32}(\mathrm{~d} / 8 \mathrm{kpc})^{2} \mathrm{erg} \mathrm{s}^{-1}$. Then XMMU J174554.4-285456 is a transient source detected here, for the first time, during an outburst on October 3, 2002, ruling out for example the outburst of a supernova. Such very rapid luminosity decrease is not compatible with a tidal disruption of a star as observed in some non active galaxies (e.g., Halpern et al. 2004). The X-ray properties suggests an X-ray binary nature, involving a compact object which could be either a white dwarf, or a neutron star, or a black hole.

The typical outburst luminosity of white dwarf system is only up to about $10^{34} \mathrm{erg} \mathrm{s}^{-1}$ (e.g., GK Per, Sen \& Osborne 1998), implying a distance upper limit of about $2.5 \mathrm{kpc}$, and the bulk of the observed hydrogen column density would be for instance intrinsic, produced by the accreting material. However, here the amplitude between the outburst phase and the quiescent phase is much larger than the one observed in cataclysmic variables. For example, GK Per, which has the most similar $\mathrm{X}$-ray properties (e.g., $N_{\mathrm{H}}, k T_{\text {brems }}$, period pulsation), has only an amplitude of about 10 (Hellier et al. 2004), and a significant $\mathrm{Fe} \mathrm{K} \alpha$ line is seen during outburst with an equivalent width of $160 \pm 20 \mathrm{eV}$ typical for cataclysmic variables (see Ezuka \& Ishida 1999). Therefore, a cataclysmic variable origin is very unlikely for XMMU J174554.4-285456.

The companion of the neutron star or of the black hole can be either a low mass object or a high-mass object. A subclass of low mass X-ray binaries are transient systems, called $X$-ray novae, which undergo sometimes outbursts. For most of the time, X-ray novae are in quiescent state, where the mass accretion rate from the disk to the compact object is very small, producing a low-level of X-ray emission. The quiescent $0.5-10 \mathrm{keV} \mathrm{X}$-ray luminosities of neutron star $\mathrm{X}$-ray novae are about $10^{32}-10^{34} \mathrm{erg} \mathrm{s}^{-1}$, i.e., 100 times higher than those for black hole X-ray novae $\left(\sim 10^{30}-10^{33} \mathrm{erg} \mathrm{s}^{-1}\right)$, as shown by Garcia et al. (2001). Here we find an upper limit of the luminosity in the quiescent state of $1.2 \times 10^{32}(d / 8 \mathrm{kpc})^{2} \mathrm{erg} \mathrm{s}^{-1}$, i.e., compatible with a black hole X-ray nova or a neutron star X-ray nova. The lower limit amplitude between the outburst phase and the quiescent phase is consistent with both a neutron star and a black hole (Chen et al. 1997). Therefore XMMU J174554.4-285456 can be either a neutron star X-ray nova or a black hole X-ray nova. XMMUJ174554.4-285456 can also be a high mass $\mathrm{X}$-ray binary formed by a neutron star or a black hole and a primary Be star companion. Indeed, as reported by Liu et al. (2000), most of the transient high mass X-ray binaries are Be star systems. Roughly two-third of a sample of 130 high mass X-ray binaries are $\mathrm{Be} / \mathrm{X}$-ray binaries, and X-ray pulsations have been found in about $60 \mathrm{Be} / \mathrm{X}$-ray binary systems (Ziólkowski 2002).

We conclude that a higher $S / N$ X-ray observation of XMMU J174554.4-285456 during its outburst phase is needed to confirm or reject the candidate X-ray pulsation at $172 \mathrm{~s}$ suggested by our timing analysis. If confirmed, we will be able to firmly identify the compact object as a neutron star. Moreover near-infrared follow-up observations are needed to identify and to characterize the companion star.

Acknowledgements. This work is based on observations obtained with $X M M-N e w t o n$, an ESA science mission with instruments and contributions directly funded by ESA Member States and the USA (NASA). D.P. is supported by a MPE fellowship.

\section{References}

Andronov I. L. 1994, Odessa Astron. Publ., 7, 49, http://il-a.pochta.ru/oap7_049.ps

Chen W., Shrader C. R., \& Livio M. 1997, ApJ, 491, 312

Churazov, E., Gilfanov, M., Sunyaev, R., et al. 1997, Adv. Space Res., 19,55

Ezuka H., \& Ishida M. 1999, ApJS, 120, 277

Garcia M. R., McClintock J. E., Narayan R., et al. 2001, ApJ, 553, L47

Halpern, J. P., Gezari, S., \& Komossa, S. 2004, ApJ, 604, 572

Hellier C., Harmer S., \& Beardmore A. P. 2004, MNRAS, 349, 710

Liu, Q. Z., van Paradijs, J., \& van den Heuvel, E. P. J. 2000, A\&AS, 147,25

Lomb N. R. 1976, Ap\&SS, 39, 447

Mitsuda, K., Inoue, H., Koyama, K., et al. 1984, PASJ, 36, 741

Muno, M. P., Baganoff, F. K., \& Bautz, M. W. 2003, ApJ, 589, 225

Porquet, D., Rodriguez, J., Corbel, S., et al. 2003a, A\&A, 406, 299

Porquet, D., Predehl, P., Aschenbach B., et al. 2003b, A\&A, 407, L17

Predehl, P., \& Schmitt, J. H. M. M. 1995, A\&A, 293, 889

Rieke, G. H., Rieke, M. J., \& Paul, A. E. 1989, ApJ, 336, 752

Sakano, M., Koyama, K., Murakami, H., et al. 2002, ApJS, 138, 19

Sakano, M., Warwick, R. S., Hands, A., \& Decourchelle, A. 2004, MmSAI, 75, 498

Scargle J. D. 1982, ApJ, 263, 835

Sen C., \& Osborne J. P. 1998, ASP Conf. Ser., 137, 463

Sidoli, L., Mereghetti, S., Israel, G. L., et al. 1999, ApJ, 525, 215

Wilms, J., Allen, A., McCray, R. 2000, ApJ, 542, 914

Ziólkowski J. 2002, Mem. Soc. Astron. It., 73, 1038 